\title{
Approche anthropologique de la violence structurelle en vue du développement économique des territoires
}

Violence and territorial development: anthropological in a worldwide competitive economy

Gewalt und territoriale Entwicklung: anthropologischer Ansatz in einer weltweit wettbewerbsfähigen Wirtschaft

\section{Anthony Tchékémian et Richard Gauthier}

\section{OpenEdition \\ Journals}

Édition électronique

URL : http://journals.openedition.org/rge/2890

DOI : $10.4000 /$ rge.2890

ISSN : $2108-6478$

Éditeur

Association des géographes de l'Est

Édition imprimée

Date de publication : 31 octobre 2010

ISSN : 0035-3213

\section{Référence électronique}

Anthony Tchékémian et Richard Gauthier, « Approche anthropologique de la violence structurelle en vue du développement économique des territoires », Revue Géographique de l'Est [En ligne], vol. 50 / 1-2 | 2010, mis en ligne le 06 septembre 2011, consulté le 08 septembre 2020. URL : http:// journals.openedition.org/rge/2890; DOI : https://doi.org/10.4000/rge.2890

Ce document a été généré automatiquement le 8 septembre 2020

Tous droits réservés 


\title{
Approche anthropologique de la violence structurelle en vue du développement économique des territoires
}

\author{
Violence and territorial development: anthropological in a worldwide \\ competitive economy \\ Gewalt und territoriale Entwicklung: anthropologischer Ansatz in einer weltweit \\ wettbewerbsfähigen Wirtschaft
}

Anthony Tchékémian et Richard Gauthier

\begin{abstract}
« Nous aurons raison de tout. Et d'abord nous ruinerons cette civilisation qui vous est chère, où vous êtes moulés comme des fossiles dans le schiste. Monde occidental, tu es condamné à mort. Nous sommes les défaitistes de l'Europe, prenez garde, ou plutôt non : riez encore. Nous pactiserons avec tous vos ennemis, nous avons déjà signé avec ce démon le Rêve, le parchemin scellé de notre sang et de celui des pavots. Nous nous liguerons avec les grands réservoirs de l'irréel. Que l'Orient, votre terreur, enfin, à notre voix réponde. Nous réveillerons partout les germes de la confusion et du malaise. Nous sommes les agitateurs de l'esprit. [...] Juifs, sortez des ghettos [...] Bouge, Inde aux milles bras, grand Brahma légendaire. A toi, Egypte [...] Soulève-toi, monde [...] Riez bien. Nous sommes ceux-là qui donneront toujours la main à l'ennemi. ».

Louis Aragon (1925)
\end{abstract}

\section{Introduction}

1 A l'occasion de ce colloque sur le thème atypique « Firmes, géopolitique et territoires ", l'étude du monde des firmes et leurs comportements sur les territoires, conduit à une approche anthropologique, voulue novatrice, en raison de son caractère de pluridisciplinarité, au travers de la géographie, de l'économie, et de l'histoire du droit. 
2 Une analyse du contexte d'insertion des firmes sur les territoires conduit à une approche anthropologique, voulue novatrice, en raison de son caractère pluridisciplinaire. La pertinence du sujet s'affirme au vu des concepts modernes de mondialisation, de libre échange, qui sont souvent la cause de rapports de force, de violence. Les conditions de pauvreté dues au développement du commerce international et l'implantation industrielle de firmes sur les territoires créent une violence structurelle. Nous limiterons notre propos à cet aspect, en écartant les agressions des populations locales contre les représentants des firmes. La violence ethnique n'est pas propre à la globalisation: elle existait déjà durant la période coloniale, et avant. Aucun jugement ne sera porté sur les pratiques des firmes, ni sur celles ancestrales, des peuples autochtones, mais nous montrerons l'intérêt de réunir différentes disciplines, pour œuvrer au développement et à la prospective territoriale.

3 Le géographe doit reformuler d'anciens cadres d'analyse, loin des présupposés théoriques des économistes, motivés par la maximisation du profit. Il y a bien un rapport au territoire et au temps qui s'établit entre les firmes qui viennent s'installer et les sociétés déjà en place. La géographie permet d'analyser ces rapports, mais les outils de l'anthropologue sont déterminants pour les comprendre. La référence à la temporalité mérite intérêt, car nous sommes dans un temps où le rapport des populations au passé a évolué : "un peuple ne peut plus se définir comme authentique ou traditionnel, à moins de verser dans l'idéologie politique. Tous les produits culturels sont aujourd'hui le résultat de parodies, de pastiches, de collages. Chacun joue avec les valeurs du passé, dans un va-et-vient permanent avec le présent » (Abeles, 2008).

4 La perte des mythes, à notre époque, peut être la cause d'une crise de comportement social, et influence l'inconscient collectif. Les sciences sociales ont mis en évidence cet aspect. Au VIII AC, selon Ovide, "L'âge d'or commença. Alors les hommes gardaient volontairement la justice et suivaient la vertu sans effort. Ils ne connaissaient ni la crainte, ni les supplices; des lois menaçantes n'étaient point gravées sur des tables d'airain [...] 》 (Ovide, 2005). Il y aurait eu autrefois dès l'Antiquité, un âge d'or, un paradis terrestre. Ovide s'inspire de théories mythologiques et philosophiques, avec en toile de fond, la question chrétienne, qui culpabilise l'homme par le péché originel. Ceci a été développé par Girard (1986) qui vise à trouver le principe explicatif de la diversité des cultures. La théorie de Girard a révélé le secret caché de la violence au cœur de toute civilisation : "C'est pour expulser la vérité au sujet de la violence qu'on se confie à la violence». Ainsi, la violence est immanente, elle est intégrée au territoire du fait de la présence d'un dieu vengeur, celui du monothéisme chrétien.

5 Malinowski est connu pour avoir systématisé la pratique de l'anthropologie de terrain et sa méthode d'observation participante. A partir de 1913, il travaille de nombreuses années en Nouvelle-Guinée, chez les Mélanésiens des îles, et analyse le système d'échange de la Kùla ${ }^{1}$. Puis, il s'intéresse à la violence dans les sociétés primitives et montre le lien avec le territoire, ainsi que les mécanismes de conflits dans les groupes (Malinowski, 2001). Horchani (2006) met en évidence la question du désordre mondial et ses conséquences sur les territoires avec pour solution la recherche d'équilibre (Horchani, 2006), qui est donc l'antithèse de la crise, apparentée à un déséquilibre. L'ensemble de ces sources montre une convergence vers la prise en compte des comportements humains sur un territoire donné. La nécessité d'une protection durable apparait. La dimension humaine est délaissée par la théorie économique du libre échange. Trois observations peuvent être induites de ces travaux scientifiques. 
6 La première consiste à dire que "les critères du droit international sont flous » (Cot, 2006) : ce constat est dangereux, car en observant les enjeux de Copenhague de décembre 2009, il peut être souligné les accords internationaux d'élargissement du système de droits à polluer, accordés aux pays en développement, qui sont complétés par les garanties d'une assistance technique et financière. Il s'agit en fait d'une véritable négociation financière de "droits à polluer ». Les critères américains sont énoncés par le préteur («judge made law») : c'est le juge qui fait la loi, et non le législateur, les minorités, le peuple ${ }^{2}$. Millard (2000) conclut à un « réalisme juridique » (Millard, 2000) ${ }^{3}$. Delmas-Marty (2004), spécialiste de la mondialisation du droit, a une vision globale des problèmes, et conclut à l'imperfection du droit mondial : le droit international n'est pas cohérent, et n'est pas un édifice parfait ${ }^{4} \mathrm{Il}$ y a une dialectique entre le droit et la coutume, qui est un usage répété dans le temps : le droit mondialisé reste théorique et abstrait, la coutume demeure plus flexible, plus adaptée au territoire.

7 La seconde observation est que l'œil occidental moderne a en face de lui la diversité des territoires traditionnels. Il s'agit d'une représentation du réel : l'occidental a une vision et une position ethnocentriste et voit ces problèmes à travers sa propre conception des territoires extérieurs. Il apparait une opposition entre l'état civilisé et les Inuits, ce qui révèle un jugement de valeur implicite : d'une part, il existe des territoires développés, et d'autre part des territoires primitifs. Malaurie montre le sort des Inuits, leur mode de survie ou de disparition (Malaurie, 2008); par le destin tragique de ces peuples, notre incapacité à gérer la planète apparait à nos yeux d'homme, présumés civilisés.

Levi-Strauss présente un ouvrage au titre évocateur, Le regard éloigné, dans lequel il montre la distance entre notre civilisation et le regard que l'on porte sur les territoires, lieu de libres échanges, dans le cadre de la mondialisation et nous incite à des réflexions sur la valeur des cultures, sur la place accordée à l'autre, et sur la liberté. En travaillant sur les mythes et les structures inconscientes des sociétés, il élabore une approche où universalisme et différence ne sont pas contradictoires (Levi-Strauss, 1983)5. Levi-Strauss rejette les démarches ethnologiques qui « rangent les peuples étudiés dans des catégories séparées de la nôtre, les mettant au plus près de la nature, comme l'implique l'étymologie du mot sauvage [du latin silva, forêt] et, de façon plus explicite, l'expression allemande Naturvölkern; ou bien hors de l'histoire quand elle les dénomme primitifs ou archaïques, ce qui est une autre façon de leur refuser un attribut constitutif de la condition humaine " (Levi-Strauss, 2007). Il récuse la notion implicite et prégnante "d'un progrès continu le long d'une route sur laquelle l'Occident seul aurait brûlé les étapes $»^{6}$. A ses yeux, les modes de pensée des sociétés dites "primitives » ne se distinguent pas par leur degré de rationalité. La pensée sauvage, expression qu'il utilise pour décrire la pensée à l'état brut, naturel, étant "logique, dans le même sens et de la même façon que la nôtre " ${ }^{7}$. Son examen de la magie et du mythe renverse les perspectives : alors que l'universalisme occidental était jusqu'alors sous-tendu par l'idée du progrès linéaire et continu.

9 Morgan, fondateur de l'anthropologie politique, pré-marxiste, s'intéresse aux confédérations indiennes, et innove par sa méthodologie : il vécut parmi les Iroquois et décrit leur vie sociale et culturelle, faisant de sa propre expérience le matériau brut de sa réflexion. Il développe la théorie évolutionniste dont il fut le plus fervent défenseur, en avançant l'idée que l'évolution de l'humanité suit un schéma unique, caractérisé par trois stades successifs observables sur un territoire : la sauvagerie, la barbarie et la civilisation (Morgan, 1985). 
10 La troisième observation provient de Nader (1969) connu pour ses avancées significatives en matière d'analyse des conflits, et qui pratique l'analyse processuelle (Nader, 1978). Cette méthode scientifique permet de comprendre et d'analyser un territoire, en s'attachant aux processus conflictuels.

11 Dans le langage moderne apparait l'expression « développement durable », qui montre la nécessité d'observation à très long terme (trois cents, cinq cents ans) car épuiser les ressources cause la fin de l'histoire. Cette remarque s'inscrit dans les réflexions actuelles des géographes prétendent que le développement durable est «un instrument d'émancipation" (Berdoulay, 2000) qui étant admis que les Nations unies s'attachent à protéger les revendications et les droits des peuples autochtones ${ }^{8}$. La démarche aménagiste pour être transposée implique une synthèse entre des solutions particulières, expérimentées et des modèles généraux, probants. Il existe un subtil jeu de miroir entre l'expérience aménagiste dans les colonies et les enjeux actuels du développement durable. L'originalité de cette approche repose sur la nécessité du détour colonial pour enrichir les débats sur la question environnementale et l'aménagement. Marx dans son œuvre Le capital, justifie l'interrogation sur l'épuisement des ressources naturelles en affirmant que sur un territoire existe une loi de baisse tendancielle du taux de profits : "sur un territoire, le travail est subordonné au capital » (Marx, 1968), donc à la finance.

12 Cette problématique de recherche nous conduit à présenter un plan en deux parties. La première a trait à la relation qui s'instaure entre la notion de territoire et les différentes formes de conflits. Les influences respectives des traditions et des formes de violence seront exposées. La seconde partie sera consacrée à l'exposé des théories du don, antidote de la violence au sein des territoires, ce qui conduira à présenter les anciens systèmes d'échange et de don, et la théorie spécifique des prestations totales, système de prestation de paix basé sur l'échange réciproque. La compréhension du domaine du territoire conduit à une approche par l'irrationnel : le phénomène de la violence côtoie le domaine mythique, et son approche est anthropologique.

\section{Territoires et conflits}

13 L'anthropologie a centré ses analyses des territoires sur la question des conflits, car l'histoire humaine révèle des pratiques anthropophages: telles celles des Maoris décrites par Sahlins (2003). De tels exemples peuvent être multipliés et la violence est immanente sur un territoire. Il s'agirait d'une forme de loi générale fondée sur des traditions ancestrales, étudiée en premier lieu, ce qui confère à la violence un rôle géoéconomique évident au sens de Luttwak (1994) et de Lorot (2000), aspect développé en second lieu.

\section{A. Le poids des traditions}

Les observations des anthropologues montrent que dans l'ordre idéal, les territoires dits sauvages, sont des lieux de sagesse; l'œil occidental, ne peut plus afficher de mépris à leur égard. L'adage est que toute terre ne doit pas sortir du groupe parental (Verdier, 1971) : il y a un lien ancestral entre le groupe humain et la terre, ce qui est le cas chez les amérindiens et les esquimaux. L'homme est éphémère, la vie sociale dépend de mythes : il existe le désordre dans les croyances, ce que les hommes et les 
dieux doivent combattre. En substituant le mot crise à celui de désordre, il apparaît que celle-ci est de la responsabilité des dieux et des hommes. En tant que forme de désordre, la notion de crise est ancienne : "la loi est mythique, la coutume est ancestrale " (Rasmussen, 1931). Les expéditions chez les esquimaux révèlent un usage : «nous faisons ainsi parce que nos parents l'on toujours fait " (Rasmussen, 1931). Les conflits sont réglés par les anciens, les notables en liaison avec les esprits ancestraux. La dimension du mythe prend tout son sens. Chez les Agni, le rapport s'inverse: "la terre possède l'homme» (Rouland, 1995); le territoire possède l'homme.

Il existe un équilibre de l'échange entre le groupe et l'individu, et il s'agit de communautarisme. Dans la vallée du Nil, après la mort, les ancêtres se réincarnent, l'individu et son esprit sont animés d'une force vitale (Diaw, 1863). La terre, le territoire sont des propriétés communautaires et non privées, avec des droits individuels; des conséquences apparaissent :

- la terre est exo-intransmissible : elle ne se transmet pas à l'extérieur, ce qui constitue un interdit, sauf lors de cession temporaire ou précaire ;

- l'endo-aliénation est possible à l'intérieur du groupe : il peut y avoir échange sur la terre au sein d'un même groupe, mais la valeur est minorée, et la terre perd de sa valeur ;

- les pactes, les contrats, les accords, les engagements sont influencés par des puissances invisibles : il s'agit de l'aspect irrationnel du droit, ce n'est pas un droit écrit. La suppression de comportements coutumiers est source de conflits, d'échecs, par exemple lors de la colonisation en Algérie, Indochine, Indes, Madagascar. Les alliances fécondatrices se font avec Dieu, ce qui engendre la vie, et cette croyance est source de fécondité. A contrario, si celle-ci n'a plus cours le territoire meurt. Les firmes doivent prendre en compte les cultures, les croyances au sein des territoires. Par ailleurs, des alliances fondatrices avec Dieu jouent un rôle majeur et influence des chefferies qui apparaissent, caractérisées par deux aspects (Rouland, 1995) : le chef de terre, de territoire, a un pouvoir sur les hommes, comme au Sénégal au sein de la communauté Toubab, communauté Maures ; le chef de lignage impose l'exo-intransmissibilité des terres lignagères, aussi le territoire est donc lignager. Dans ces sociétés patriarcales, les femmes sont exclues de la succession à la terre. Le pouvoir appartient aux hommes, ce qui montre le poids des traditions, et justifie les mouvements féministes.

\section{B. Le poids de la violence}

Ce point met en évidence les perspectives de l'anthropologie moderne de la violence, notamment la question du transfert des systèmes culturels, juridiques et politiques, ce qui fût pratiqué dans les hypothèses coloniales, et se généralise dans le contexte de la mondialisation.

Les premières études datent des années cinquante, menées par l'Institut Max Planck qui s'intéresse à la physiologie du comportement, et qui donne lieu en 1973 à l'obtention d'un prix Nobel décerné à trois lauréats : Von Frisch, Timbergen, et Lorenz. En 1960, ces chercheurs s'intéressent aux structures neuromotrices, à la maturation et mettent en évidences un concept majeur, celui de «l'évolution régressive ». Ainsi, le processus d'hominisation se caractérise par une évolution régressive, nous constatons l'existence de cycles de violences et au lieu d'évoluer vers l'intelligence, la convergence, l'homme est victime de son autodestruction. Cette vision psychanalytique peut être dépassée par la perspective ouverte par Teilhard de Chardin : "Tout en vous maintenant 
sur votre ligne propre, montez vers toujours plus de conscience et d'amour. Au sommet, vous vous trouverez réunis à ceux qui, de divers côtés, auront entrepris une ascension semblable. Car tout ce qui monte converge. " (Teilhard de Chardin, 1947).

A titre d'illustration, diverses pratiques coutumières violentes ont eu lieu au cours des siècles :

- au V AC, les anciens germains, à l'époque franque, pratiquaient la vengeance privée, nommée faïda : elle était pratiquée dans les cas les plus graves, tels que les homicides, incendies, rapts de femmes, et à condition que cette vengeance n'aille pas à l'encontre de la paix royale (Balon, 1965) ;

- jusqu'au XIII PC, la magie païenne justifie la pratique des ordalies : cet ancien mode de preuve en justice, de nature religieuse, est aussi appelé jugement de Dieu. Il consiste à soumettre les plaidants à une épreuve de l'eau, du fer et du feu, dont l'issue, déterminée par Dieu, désigne le coupable, ce qui sera interdit par le Concile de Latran en 1215 et par Saint Louis en 1258 qui promulgue un édit et dispose en remplacement le serment purgatoire et le témoignage oral (Carbasse, 2000) ;

- Robespierre, suite à la loi du 10 juin 1794, 22 prairials An II, supprime les ennemis du peuple et dispose : Article $4:$ : Le tribunal révolutionnaire est institué pour punir les ennemis du peuple. »; Article $5:$ : Les ennemis du peuple sont ceux qui cherchent à anéantir la liberté publique. "; Article 6 : «Sont réputés ennemis du peuple, ceux qui auront provoqué le rétablissement de la royauté, ou cherché à avilir ou à dissoudre la Convention nationale et le gouvernement révolutionnaire et républicain. [...] Ceux qui auront cherché à empêcher les approvisionnements de Paris, ou à causer la disette dans la République. ";

- au XIX PC, lors de la colonisation et de ses excès, comme à Madagascar, au Congo, en Indochine ;

- au XX PC, à l'occasion des violences nazies, fascistes, staliniennes, chinoises, cubaines.

Par ces exemples, le phénomène violent apparaît clairement dans sa dimension historique et traditionnelle, et il a tendance à se généraliser à l'époque contemporaine, à se mondialiser (terrorisme, prises d'otage, sabotage d'infrastructures).

Pour compléter la réflexion, il convient de présenter les doctrines anthropologiques modernes qui éclairent la question fondamentale de l'échange et celle des mécanismes de transferts de systèmes culturels, juridiques et linguistiques.

\section{Les doctrines anthropologiques modernes}

21 Développée par Verdier (1984), deux constats sont avancés, qui peuvent former une hypothèse et un cadre conceptuel : le premier, est que sur un territoire donné, toute loi sociale est une loi d'échange ; le second, la compréhension de la violence se fait à partir de dettes respectives. Sans porter de jugement, on constate deux cas différents de comportements de vengeance :

- le premier, en Ethiopie, chez les Gamo, la vengeance est interdite car elle est jugée trop dangereuse, pour l'individu, le groupe et le territoire ;

- le second consiste en un exemple de régression, dans le Caucase, le sang ne vieillit pas : s'il y a eu un homicide, la vengeance s'exerce immédiatement et se perpétue de façon interminable de génération en génération. Pour les Abkhazes, un dicton local dit : « nous ne faisons pas commerce du sang de nos frères ", autrement dit un contre-don ne peut pas stopper la vengeance. 

est-ouest ", le pipeline Bakou-Tbilissi-Ceyhan, nous donne l'occasion de rappeler qu'a eu lieu un sabotage en septembre 2008, que le 15 juillet 2009 est assassiné la journaliste indépendante Natalia Estemirova puis son avocat qui dénonçaient les pratiques des trusts Russes. L'ONG Mémorial l'a soutenu, parlant "des émirats du Caucase », donc d'une fortune commerciale et industrielle qui justifie les déplacements des populations, dont certaines émigrent en France. Cet exemple prouve que sur le territoire, au nom de l'approvisionnement économique pétrolier, on tue encore de nos jours. tendent à écraser une communauté, et à déstructurer un territoire, ce qui traduit un fonctionnement pathologique. Ainsi, en Ossétie, le meurtrier entre comme fils adoptif dans la famille adverse. En droit, il s'agit d'abandon noxal.

Après la description de la pathologie de la violence, on peut se poser une question : la fin de la violence est-elle symbolique, par un don de vie, un sacrifice, appelée " composition ", au sens de transaction? Par exemple, chez les Moundang, il existe un rite de réconciliation : on sort de la violence en tuant un animal domestique, le chef de lignage baigne ses mains dans le sang de la bête, puis se les lave ${ }^{9}$. Autrement dit, on offre la mort pour une vie. Ainsi, le droit barbare, vu précédemment, survit. La subtilité réside dans le fait que dans le premier cas on parle de coutume, alors que dans le second, il s'agit de droit.

Autre exemple, dans le détroit Behring, chez les Inuits, se produisent de violents conflits, des massacres de captifs, observés par Malaurie $(1974)^{10}$. Celui-ci a également joué un rôle déterminant, de témoin-expert dans le procès engagé près la Haute Cour danoise, quant à l'illégalité de leur expulsion (exil forcé et droit au retour sur plus de $3000 \mathrm{~km}^{2}$ de territoire de chasse et de pêche) suite à l'installation de la base militaire américaine de Thulé. En outre, le 21 janvier 1968, eut lieu le crash d'un avion américain de la base de Thulé, avec quatre bombes nucléaires à bord ${ }^{11}$. Trois bombes ont été récupérées, la dernière est immergée en pleine mer entrainant des dommages sur les hommes et l'environnement (Sagan, 1993).

Les observations fournies par les doctrines modernes sont l'occasion de souligner l'aspect pathologique et généralisé de la violence et ses répercussions sur les territoires où s'implantent firmes et institutions. D'autres exemples, moins confidentiels, peuvent être cités mais en nous limitant à celui-ci nous soulignons le caractère dommageable d'une telle implantation qui a privé pendant plus de cinquante ans les esquimaux de leur territoire, par un exil forcé. La multiplication de telles situations n'est-elle pas de nature à fonder une véritable loi générale d'intolérance institutionnelle de rejet de l'altérité et de négation d'autrui ? La question des transferts culturels se trouve donc posée dans sa dimension humaine, juridique.

\section{Les mécanismes de transferts de systèmes}

27 L'analyse systémique montre un état de multiplication des situations de transferts, de méthodes juridiques et économiques de territoire à territoire, amplifié par la mondialisation. La procédure d'acculturation se caractérise par le changement structurel, la déculturation, l'assimilation. Cela permet de mettre en exergue cinq éléments d'analyse systémique autour des transferts.

Revue Géographique de l'Est, vol. 50 / 1-2 | 2010 

quasi-servitude, rapport d'un territoire dominant à un autre, dominé (ainsi en Arctique, Irak, Afghanistan, Koweït, Algérie, Cote d'Ivoire, Afrique du Sud, Colombie, Haïti, Caucase, Ukraine, Palestine, Tibet, Corée). Sur ces territoires, une culture tend à être imposée, au nom d'intérêts dominants soutenus par des implantations commerciales, financières ou militaires. Les transferts de systèmes modernes sont impossibles dans un territoire traditionnel. Certes cela ne convainc pas tout le monde, notamment les américains qui considèrent que leur modèle culturel peut être appliqué partout, au nom de la démocratie. Par exemple, la ville minière d'Atlanta, ce nouvel émirat pétrolier se situe au nord du Canada, au pays des Indiens Cree, des lacs et des élans, zone de sables bitumineux d'Alberta où se trouve le deuxième gisement pétrolier du monde après l'Arabie Saoudite. Son exploitation reste difficile et nécessite l'emploi de solvants naphta (Shields, 2009). Une obligation de mutation du territoire apparaît, qui se heurte à la résistance des institutions communautaires, qui se chargent d'attribuer les territoires, de répartir les terres.

Le second à pour sujet l'islamisation des territoires de droit coutumier, comme au Niger, en Mauritanie, au Sénégal, en Guinée, au Mali, ce qui a donné lieu à un article qui dénonce "la résistance du droit africain à la modernisation " (Blanc-Jouvan, 1977). Les systèmes antéislamique pratiqués par les bédouins révèlent une forme de communautarisme et se fondent sur un droit oral.

30 Le troisième élément d'analyse met en évidence le fait que le droit moderne se substitue au droit traditionnel, mais le droit familial est peu touché, par exemple, l'institution du sororat ${ }^{12}$ consiste pour un veuf à épouser une sœur de son épouse, en particulier lorsque cette dernière laisse des enfants en bas âge. Les anglais réduisent la pression sur les traditions et évitent les conflits par la common law ${ }^{13}$ en se bornant à réinterpréter les coutumes étrangères. Les français, et en particulier Ernest Roume (1853-1941), gouverneur général de l'Afrique Occidentale Française de 1902 à 1908, a ambitionné de rédiger un coutumier général. L'œuvre est poursuivie par son successeur, Jules Brévié, gouverneur en 1936, lequel échoue face à ce colossal travail (Leroy, 1982).

31 Le quatrième aspect est celui de la Chine ancienne qui déteste les codifications, tout comme Mao : il s'agit de conclusion d'études de théoriciens chinois qui ont mis au point cette distinction linguistique. Le " $\mathrm{Li}$ » qui est la pensée de Mao, le guide suprême associé aux décisions du Partie Communiste Chinois (PCC) ; et le «Fa» qui en droit pénal est réservé aux étrangers et aux contres révolutionnaires. Un texte de Confucius bien connu des lettrés chinois dispose (Tay, 1998) : «Si le peuple est assujetti aux lois et si l'uniformité est imposée au moyen de sanctions, les gens chercheront à s'y soustraire et ne connaitront pas la honte, alors que si le peuple est gouverné par la vertu et que l'uniformité est recherchée au moyen des $L i^{14}$ les gens auront un sentiment de honte et deviendront par conséquent justes. $\aleph^{15}$. Cette citation dévoile un principe fondateur de l'éthique chinoise : la morale (Mao, 1972) prime le droit. La pensée traditionnelle chinoise estime qu'un constant recours au «fa », ou droit positif, est la preuve d'un effondrement de l'ordre social et d'un manque d'harmonie entre l'Etat et la société.

32 Le cinquième élément d'analyse, date de 1865 , il s'appuie sur l'application de l'arrêté Faidherbe, qui dispose le droit éminent de l'état français à l'aménagement du territoire, ce qui atteste de la perspective coloniale : les territoires sont définis en droit comme des terres vacantes et sans maître. Ainsi, la maîtrise du sol échoit exclusivement à l'état 
colonial. Pour la tradition, les droits sur la terre sont collectifs, les droits sur les cultures appartiennent aux individus. Des faits illustrent cette répartition des droits : en 1964, le Sénégal crée un domaine national et consent des usufruits aux conseils de communautés; en 1974, le Togo constitue un domaine foncier national sans accaparement des terres incultes qu'il redistribue; en 1977, le Bénin nationalise le sol (Verdier, 1962). Refuser d'intégrer les mentalités, les besoins des populations autochtones en cas de mise en œuvre de nouvelles politiques de développement, accroit la résistance des systèmes traditionnels (Poirier, 1956).

Ces cinq éléments d'analyse montrent qu'éradiquer la coutume bouleverse les sociétés traditionnelles, qui se défendent contre ce type de procédures, perçues comme agressives. Ce qui peut, dans certains cas, déclencher des conflits et une violence collective. Les sociétés de tradition ne sont pas uniformes, elles sont pluralistes et incitent le législateur à réduire les prétentions d'état au monopole sur un territoire donné. Le droit traditionnel est flexible, mais on ne le perçoit pas comme tel. Il heurte les juristes habitués à un droit de sanction, répressif. Seule la loi peut équilibrer le droit de l'état et celui des communautés (Alliot, 1965 a et b).

\section{Territoire et don}

Après avoir vu que l'anthropologie avait pour objet d'analyse la question centrale de la violence, nous constatons qu'elle a développé d'importantes théories sur la question du don, de la réciprocité, de l'échange, de la gratuité, caractéristiques originales de population marquées par une sorte de communisme primitif (Mauss, 1950) ${ }^{16}$, aspects développés par nombre d'écoles.

En premier lieu, il faut souligner l'influence de Franz Boas, mathématicien et physicien, qui s'oriente ensuite vers la géographie et part en 1883 en Terre de Baffin, pour étudier l'influence de l'environnement sur le mode de vie des esquimaux. Cette expérience fait naître chez lui un intérêt pour ces peuples, jugés à l'époque, primitifs, pour leur singularité culturelle digne d'être sauvegardée. Restant très proche de la géographie, Boas se tourne vers l'anthropologie et prétend qu'il n'existe que des processus singuliers, ce qui fait de lui un relativiste. Il dissocie l'étude des races de celle des cultures, et affirme qu'aucune culture ne prime sur une autre. Boas traite chaque culture comme une synthèse originale, dotée d'un style, qui s'exprime à travers la langue, les croyances, les coutumes, l'art, pour constituer un tout (Journet, 2002). La diversité des aires culturelles susceptibles d'entrer en collision est une caractéristique majeure de la mondialisation (Huntington, 2002). Elève de Boas, tenante de l'école psychosociologique américaine, Ruth (1935), de l'université Columbia, consacre un ouvrage aux conduites chamanistiques et à l'origine symbolique d'un territoire. Sa méthode consiste en l'étude des relations entre les territoires, les cultures de groupe et, en particulier, le psychisme individuel.

En second lieu, le courant cybernétique joue un rôle fondamental dans l'innovation et la modernisation des problématiques, ce qui permet d'accroître la compréhension des sociétés en anthropologie, par l'apport d'une méthode scientifique aux domaines des sciences sociales. Cette école anglo-saxonne fut représentée par trois principaux chercheurs :

- le mathématicien Wiener (1948) révolutionne l'analyse des processus de transmission de messages dans les médias perturbés par des interférences; 
- le physicien Von Neumann en 1945 crée une nouvelle science générale du fonctionnement de l'esprit, en s'appuyant notamment sur les boucles de rétroactions, aspects propre à éclairer les travaux d'anthropologie (Faivre-Malfroy, 2009) ;

- le linguiste Chomsky (2002) fonde de nouveaux concepts : la créativité humaine, les modules mentaux, ainsi que les théories de l'émergence du langage chez l'homo sapiens. l'esprit humain. Cela est une forme de critiques des thèses de Freud, qui considérait avoir découvert l'inconscient, le moi et le sur-moi, en relation dialectique entre eux (explication des complexes, méthode hypnotique). Le courant cybernétique a cherché à comprendre les itinéraires de l'information. l'échange. Il met en évidence deux principes : d'une part, le groupe façonne l'individu ; d'autre part, l'homme est le produit de son corps. Il observe que pour progresser il faut comprendre la pensée symbolique, la magie, la notion de "mana $»^{17}$. Selon lui, la linguistique, seule, permet de comprendre les formes antérieures au contrat et à la vente (Mauss, 1950 ; Besnard, 1998 ${ }^{18}$ ).

La compréhension de la relation du territoire et du don conduit à s'attacher, d'une part, à l'étude des anciens systèmes, d'autre part à la théorie originale des prestations totales.

\section{A. La survivance des anciens systèmes}

Nos formes économiques s'expliquent historiquement sur un territoire par l'ethnographie, c'est-à-dire par l'étude des mœurs et des coutumes, puis par l'étude de l'échange dans sa composante sémitique ou gréco-romaine. Ceci conduit à diviser la notion d'échange en domaine lucratif et en domaine du don. Une bonne compréhension des doctrines anciennes impose de se référer à des sources variées : romaines, hindous et germaniques.

\section{La racine romaine}

$41 \mathrm{Au} \mathrm{V} \mathrm{AC,} \mathrm{le} \mathrm{patriciat,} \mathrm{l'aristocratie} \mathrm{romaine,} \mathrm{refuse} \mathrm{de} \mathrm{révéler} \mathrm{le} \mathrm{droit} \mathrm{par} \mathrm{écrit} \mathrm{pour}$ sauvegarder son autorité, mais en 451, la loi des XII tables (Lex duodecim tabularum) apparaît comme révolutionnaire, en ce sens que plébéiens et patriciens bénéficient alors de lois égales en matière de droit privé.

Une évolution se dessine à l'époque de Cicéron où l'on se réfère à l'Edit du prêteur (106-43 AC) qui est un véritable programme, car le juge énonce la loi.

$43 \mathrm{Au}$ VI AC, en 534, Justinien, dernier empereur byzantin, se bat pour l'unité politique impériale romaine autour de la Méditerranée. Il formule une doctrine juridique par les Institutes, et il recueille les coutumes qu'il fixe par écrit, le Digest. Il mène une politique de reconquête face aux états barbares. Ces constructions intellectuelles byzantines seront critiquées par Montesquieu, en 1748, dans De l'esprit des lois (Derathé, 1973) au motif que sur un territoire, la loi fait parler les princes comme des rhéteurs. En somme, le passage d'un droit secret au droit écrit donne aux princes le pouvoir des mots. 


\section{La théorie hindoue du don}

La théorie hindoue du don vient de l'Inde ancienne, de 1800 à 1200 AC. A cette époque, les ariens s'installent dans la zone de l'Indus, du Gange. Les textes appartiennent à la Révélation qui se nomme çruti. Elle se retrouve dans le Mahâbhârata, grand poème qui recèle des principes moraux et juridiques, traités sous l'angle narratif (Demetrian, 2005). Ainsi l'époque védique, deux siècles avant le Christ, se caractérisée par la pratique des donations royales de terres.

L'ancienne théologie brahmanique est une doctrine morale dont le précepte est: donner confère le bonheur, en ne gardant point, la richesse est faite pour être donnée.

De telles doctrines sont bien de nature à fonder une société, une civilisation, de la réciprocité, la générosité, propres à atténuer la violence, ce qui est de nature à expliquer l'œuvre immense du Mahatma Gandhi.

\section{La doctrine germanique}

Cette doctrine est étudiée par les folkloristes : c'est le gaben allemand, c'est-à-dire la générosité par le don, qui peut avoir un aspect funeste. C'est aussi une forme retrouvée dans le contrat, la vente, l'achat, le prêt, le dépôt, institutions qui entraînent la constitution de gage, autrement dit une forme de don. Il s'agit d'une tradition runique (III PC) germanique et scandinave : un principe apparait sous la forme de la circulation générale des richesses, et non de l'accaparement. De telles constatations sont de nature à expliquer les comportements collectifs modernes scandinaves, à la fois responsables, protecteurs et socialement avancés (système social, condition de la femme, style de managment, éthique politique, respect de l'environnement).

\section{B. La théorie des prestations totales}

Cette théorie prétend qu'un territoire est un marché intertribal, où s'échangent des choses, des gages de paix, des usages intertribaux, ce qui fait apparaitre à la fois un principe de rivalité, d'antagonisme, mais aussi une obligation de donner et recevoir. Dès lors, il s'agit d'un système de prestation de paix, basé sur l'échange, non monétaire. L'étude de cet aspect conduit à distinguer la dimension mythologique de la perspective morale et juridique.

\section{La dimension mythologique}

Nous pouvons constater, qu'à tort, les économistes s'attachent peu aux dimensions humaines et pacifistes, absorbés par les doctrines monétaristes, qui prennent seulement en compte les questions de masse monétaire servant d'explications fondamentales et rejetant la dimension spirituelle et mythologique.

Les travaux de Bogoras (1975) sur le détroit de Behring, au nord-est de la Sibérie, sont marquants et énoncent quatre principes qui traduisent l'importance de la dimension mythologique : échanger est vital ; les destructions sont sacrificielles ; la puissance se conquiert par le désintéressement ; les dieux sont donateurs de grandes choses.

51 A Madagascar, la famille Grandidier (1908) a étudié une ethnie, les Betsimisaraka, qui se réfèrent aux dieux créateurs, aux cultes des ancêtres, à la déesse Nemesis qui venge les 
pauvres. Il faut relever le grand intérêt des travaux des Grandidier pour les géographes et les anthropologues.

Ces ethnies sont influencées par de puissants mythes qui contribuent à leur structuration et à leur organisation. Il existe deux dimensions, l'une verticale qui donne un sens, une orientation à l'organisation sociale, l'autre horizontale, à la fois morale, éthique et juridique, qui contribue à structurer les comportements collectifs, qui n'ont de primitif que la perception qu'en ont les occidentaux. L'intérêt de l'approche anthropologique apparaît clairement à la lueur de telles observations.

\section{La dimension morale et juridique}

Un premier principe anthropologique précise que refuser un don est une déclaration de guerre, constitue une forme de théorie générale de l'obligation. Aussi, les contrats deviennent claniques, comme c'est le cas, à Bornéo, chez les populations Dayaks (Malayo-polynésiens), qui se soumettent à diverses obligations : la première est le devoir de partager le repas préparé ; la seconde est l'interdiction de refuser un viatique, sorte de dîner de voyage apportant un réconfort (Mauss, 1950) ${ }^{19}$.

Un second principe indique que tout refus d'alliance et de communication, que ce soit avec les hommes, les dieux, les esprits, conduit à la guerre, ce qui s'exprime dans divers proverbes :

\footnotetext{
- "Te ika a mani" (fidjien), "When raw it is seen when cooked it is taken", ce qui peut se traduire par : « il vaut mieux manger une nourriture à moitié cuite que d'attendre que les étrangers soient arrivés, qu'elle soit cuite et d'avoir à la partager avec eux » (Mauss, 1950). Afin d'éviter tout conflit familial, chez les fidjiens, une règle de politesse s'impose : la consommation devant les beaux-parents est prohibée (Williams, 1858).

- chez les esquimaux, les tribus indiennes Tlingit et Haïda, au cours de potlatchs, rassemblements intertribaux, il est considéré que l'esprit des morts impose aux dieux d'acheter et de rendre les choses. Une dimension spirituelle, ancestrale de l'échange non matérialiste apparaît, aspect mal perçu par les occidentaux ; ce que précise MAUSS (1924) : " (...) lors de la fête des mila-mila ${ }^{20}$, potlatch en l'honneur des morts, les deux sortes de vaygu'a, ceux $d u$ kula et ceux que Malinowski appelle pour la première fois ${ }^{21}$ les "vaygu'a permanents ", sont exposés et offerts aux esprits sur une plate-forme identique à celle du chef. Ceci rend leurs esprits bons. Ils emportent l'ombre de ces choses précieuses au pays des morts ${ }^{22}$, où ils rivalisent de richesses comme rivalisent les hommes vivants qui reviennent d'un kula solennel ». Ainsi, les choses précieuses ont une ombre au pays des morts, ce qui s'apparente à la démarche de la mythologie grecque. Aussi, les firmes, en extrayant les ressources d'un territoire, n'emportent pas d'authentiques valeurs.

- pour les Haoussa, groupe du Tchad et du nord Nigéria, islamisés, tout don se destine aux enfants et aux pauvres. Nous trouvons là, un fondement d'une théorie de l'aumône, forme du droit, morale du don qui créée une obligation.
}

L'actualité est chargée d'exemples qui illustrent toutes ces observations. France Culture, le 25 septembre 2010, dans son émission «Terre à terre ", sensibilise l'opinion sur la question de la condition de vie du peuple Kichwa d'Amazonie équatorienne, qui sont chassés de leurs territoires par les compagnies pétrolières qui exploitent les ressources présentes dans la forêt (bois, pétrole) et engendrent un désastre écologique et humain dans la région ${ }^{23}$. 
Ces deux principes mettent en évidence un corpus éthique, moral élaboré lentement par addition d'usages, de coutumes, sorte d'agrégation de comportements, qui permet d'induire une règle de conduite collective. Une telle pratique se situe aux antipodes des institutions modernes qui procèdent en élaborant, d'abord, un corps de règles théoriques, pour ensuite, les appliquer sans distinction (par exemple: codification mondiale du droit, Delmas-Marty, 2004).

\section{Conclusion}

57 Les conceptions d'économie, de marketing, de management, sont parfois étroites et mettent de côté l'approche de l'anthropologie géographique, nécessairement plus ouverte, puisqu'orientée vers des comportements du groupe mus par des mythes, des usages ancestraux qui sont la source d'une structure non primitive et évolutive. En matière de territoire seul le raisonnement à long terme mérite intérêt afin de sauvegarder la durabilité des éléments constitutifs d'un écosystème. Cela impose un devoir éthique aux opérateurs économiques qui s'implantent sur un territoire étranger, qui doivent protéger leur environnement, au nom du principe de sauvegarde du "patrimoine de l'humanité ", car finalement deux générations ont un droit égal à l'existence du milieu naturel. Tout ceci conduit à arbitrer entre une préférence pour le présent, et une pour le futur, ce qui semble bien difficile pour l'homme moderne. Il n'est pas interdit de penser, en ces matières, sur des échéances temporelles de trois cents ou cinq cents ans, pour estimer la valeur environnementale du territoire. Ce qui impose de prévoir à long terme, et d'actualiser les données sur le futur.

Le caractère restrictif des conceptions des économistes peut-être souligné, car l'« homoaeconomicus » (Makaai, 2008), l'homme économique, n'est en fait qu'un simple consommateur. Aussi, par analogie, il peut être fait référence à l'« homogéographicus ", pour signifier la prise en compte de l'homme dans son territoire, son écosystème. Ainsi, la compréhension d'un territoire astreint à prendre en compte le domaine de l'irrationnel, car la violence a incontestablement une dimension mythique et spirituelle.

\section{BIBLIOGRAPHIE}

Abeles M., 2008, Anthropologie de la globalisation, Ed. Payot, 280 p.

Adler A., 1987, « Le royaume Moundang de Léré », dans Tardits C. (dir.) Princes et serviteurs du royaume : cinq étude de monarchie africaines, Ed. Société d'ethnographie, Paris, pp. 137-170.

Alliot M., 1965, « Développement et évolution du droit privé en Afrique Noire anglophone », Les Dossiers de l'Institut des sciences juridiques du développement, Université Paris V, p. 26.

Alliot M., 1965, « Les résistances traditionnelles au droit moderne dans les Etats d'Afrique francophones et à Madagascar ", Etudes de droit africain, Cujas, Paris, pp. 235-256. 
Aragon L., 1925, « Fragments d'une conférence », parus dans La Révolution surréaliste, revue dirigée par André Breton, 15 juillet 1925, n4, p. 25.

Balon J., 1965, Traité de droit salique : étude d'exégèse et de sociologie juridiques, Ed. Les anc. Ets Godenne, Coll. Ius Medii Aevi, n³, 1244 p.

Berdoulay V., Soubeyran O., 2000, Milieu, colonisation et développement durable. Perspectives géographiques sur l'aménagement, Ed. Géographie en liberté, 254 p.

Besnard P., Fournier M., 1998, Emile Durkheim. Lettres à Marcel Mauss, Ed. PUF, coll. Sociologie, Paris, $593 \mathrm{p}$.

Blanc-Jouvan X., 1977, « La résistance du droit africain à la modernisation », Revue Sénégalaise de droit, $\mathrm{n}^{\circ} 21$, pp. 21-44.

Bogoras W., 1975, The Chukchee, New York, AMS Press, Coll. American Museum of Natural history, the Jesup north Pacific expedition, vol. 7, 733 p.

CarbasseJ-M., 2000,Histoire du droit pénal et de la justice criminelle, PUF, coll. Droit fondamental, Droit pénal, Paris, $445 \mathrm{p}$.

Chomsky N., 2002, "Perspectives on language and mind", in Belleti, A., Rizzi, L. (eds) On nature and language, Cambridge, Cambridge University Press, pp. 45-91.

Cot J-P., 2006, « Tableau de la pensée juridique américaine », dans Revue générale de droit international, Ed. Pedone, Paris, vol. 110, n³, pp. 537-596.

Delmas-Marty M., 2004, Le relatif et l'universel. Les forces imaginantes du droit, Ed. Seuil, Coll. La couleur des idées, pp. 18-23.

Demetrian S., 2005, Le Mahâbhârata, Ed. Albin Michel, Coll. Spiritualités vivantes, 569 p.

Derathé R., 1973, De l'esprit des lois, textes de Montesquieu, C-L de Secondat (trad.), Ed. Garnier, Paris, vol. 1, 566 p., vol. 2, 753 p.

Diaw Y. B., 1863, "Cahiers portant sur l'histoire des royaumes Wolof », Le Moniteur du Sénégal et dépendances comme source de l'histoire du Sénégal pendant le premier gouvernement de Faidherbe, 1856-1861, Université de Dakar.

Faivre-Malfroy F., 2009, Anthropologie de la communication, chap. 1 « La communication et la cybernétique », mis en ligne le 16 octobre 2009, consulté le 05 novembre 2010, URL : http:// www.scribd.com/doc/22652770/Anthropologie-de-la-communication-Communication-Ch-1-Lacommunication-et-la-Cybernetique

Girard R., 1986, Des choses cachées depuis la fondation du monde, Ed. LGF, Paris, 636 p.

Grandidier A., Grandidier G., 1908, Ethnographie de Madagascar, Ed. Imprimerie nationale, tome 2, p. 67.

He F., slnd, « Une longue marche vers l'Etat de Droit. La transformation du système judiciaire chinois ", Recherches en ligne, Etat de Droit et Justice, mémoire consulté le 2 novembre 2010, URL : http://www.dhdi.free.fr/recherches/etatdroitjustice/index.htm. De Bary w. M. T., 1998, Asian values and Human Rights. A Confucian Communitarian Perspective, Cambridge Mass., Harvard University Press, 196 p.

Horchani F., 2006, Où va le développement de l'investissement, désordre normatif et recherche d'équilibre, Ed. Pedone, Paris, 1 vol., 338 p.

Huntington S. P., 2002, The Clash of Civilizations and the Remaking of World Order, London, The Free Press, Simon \& Schuster, p. 367. 
Journet N., 2002, La culture. De l'universel au particulier, Ed Sciences Humaines, 384 p.

Leroy E., Wane M., 1982, « La formation des droits non-étatiques », Encyclopédie juridique de l'Afrique, vol. l'Etat et le droit, Dakar, Nouvelles éditions africaines, pp. 366-370.

Levi-Strauss C., 1983, Le regard éloigné, Ed. Plon, 398 p.

Levi-Strauss C., 2007, Tristes Tropiques, Ed. Presses Pocket, Paris, pp. 475-490.

Lévy Strauss C., 1955, Triste tropique, Ed. Plon, Paris, p. 177.

Lorot P., 2000, « La géoéconomie, nouvelle grammaire des rivalités internationales », AFRI 2000 (en ligne), Vol. 1, pp. 110-122, consulté le 22 octobre 2010, URL : http://www.afri-ct.org/IMG/ pdf/lorot2000.pdf

Luttwak E.N., 1994, The Endangered American Dream, Ed. Simon \& Schuster, 368 p.

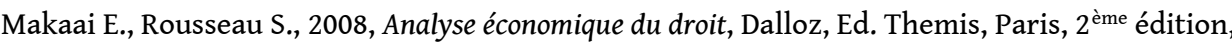
pp. 14-17.

Malaurie J, 2008, La sagesse des peuples premiers. Un recours pour notre planète, Ed. Mille et une nuits, Coll. Essais, 192 p.

Malaurie J., 1974, « Raids et esclavage dans les sociétés autochtones du détroit de Behring », Revue Inter-Nord, n¹3-14, décembre 1974, pp. 3-29.

Malinowski B., 2001, La sexualité et sa répression dans les sociétés primitives,Ed. Payot \& Rivages, Paris, $296 \mathrm{p}$.

Malinowski B., 2006, La paternité dans la psychologie primitive, Ed. L'écluse d'aval, Paris, 85 p.

Mao, 1972, Citation du Président Mao Tsetoung. Edition en langue étrangère, Pékin, p. 291.

Marx K., 1968, Le capital, Ed. Gallimard, Coll. $3^{\text {ème }}$ section, chap. 9, p. 1004.

Mauss M., 1924, «Essai sur le don. Forme et raison de l'échange dans les sociétés archaïques », dans L'Année Sociologique, seconde série, 1923-1924, p. 27.

Mauss M., 1950, Sociologie et anthropologie, « Essai sur le don. Forme et raison de l'échange dans les sociétés archaïques », Ed. PUF, Paris, pp. 144-279.

Millard E., 2000, «Legal realism and constraints », Conférence à la Faculté de droit de l'Universite de Copenhague, Danemark, septembre 2000, mis en ligne le 25 janvier 2007, consulté le 17 septembre 2010, URL : http://halshs.archives-ouvertes.fr/halshs-00126665/fr.

Morgan L.H., 1985, Ancient society, or researches in the line of human progress from savagery, through barbarism to civilization, Tucson, University of Arizona Press, 560 p.

Nader L., 1969, Law in Culture and Society, Ed. Aldine Pub. Co., Chicago, 454 p.

Nader L., Todd H.F., 1978, The Disputing Process: Law in Ten Societies, Columbia University Press, New York, $372 \mathrm{p}$.

Ovide, 2005, Les métamorphoses, Trad. et notes de A.-M. Boxus et J. Poucet, Bruxelles, livre I, pp. 89-93. Les quatre métamorphoses du monde, ou le mythe des races, pp. 89-150.

Poirier J., 1956, « L'avenir du droit coutumier négro-africain », L'avenir du droit coutumier en Afrique, Leiden, $n^{\circ} 18$, p. 168.

Rasmussen K., 1931, Intellectual culture of the Iglulik eskimos reports of the fifth Thule expedition, VIII, 1-2, Copenhagen, p. 502.

Rouland N., 1995, Anthropologie juridique. Ed. PUF, Coll. Que sais-je ?, Paris, 127 p. 
Ruth B., 1935, Patterns of culture, Routledge, London, 291 p.

Sagan S. D., 1993, The limits of safety organisations accidents and nuclear weapons, Princeton University Press, pp. 156-203. Cet ouvrage dénonce les faiblesses des organisations de contrôle et de régulation.

Sahlins M., 2003, “Artificialy maintained controversies: Global warming and Fijian cannibalism”, Anthropology Today, Blackwell, Oxford vol. 19, n³, juin 2003, pp. 3-5.

Shields A., 2009, «Le pétrole sale de l'Alberta - Portrait dévastateur d'une industrie polluante », Le Devoir.com, mis en ligne le 26 février 2009, consulté le 27 octobre 2010, URL : http:// www.ledevoir.com/politique/canada/236047/le-petrole-sale-de-l-alberta-portrait-devastateur-dune-industrie-polluante

Tay A. E-S., 1998, « Culture juridique chinoise », Une Introduction aux cultures juridiques non occidentales, Bruxelles, p. 208.

Teilhard de Chardin P., 1947, Euvres complètes.Troisième partie intitulée « Pouvoir rapprochant » et conclusion d'une conférence donnée le 8 mars 1947, Ed. du Seuil, 1959, Paris, février 1947, Tome 5, pp. 240-243.

Verdier R., 1962, Les modalités de passage de la propriété communautaire à l'appropriation privée en Afrique Noire, Cujas, Paris, pp. 140-143.

Verdier R., 1971, « Evolution et réformes foncières de l'Afrique Noire francophone », Journal of African Law, Ed. Cambridge University Press, Vol. 15, n¹, pp. 85-101.

Verdier R., Poly J-P., Courtois G., 1984, La vengeance : études d'ethnologie, d'histoire et de philosophie, Ed. Cujas, Coll. Echanges, Paris, 4 vol., 196 p.

Wiener N, 1948, Cybernetics, or control and communication in the animal and the machine, The MIT Press, Cambridge, Mass. et Wiley, New York, p. 194.

Williams T., 1858, Fiji and the fifians, London, Alexander Heylin, tome I, p. 34.

\section{NOTES}

1. «Kùla » est le nom d'un système d'échanges de biens précieux, mais sans aucune valeur économique directe pratiqué à l'est de la Nouvelle-Guinée entre une vingtaine d'îles, et décrit pour la première fois par Bronislaw Malinowski, au début du XX siècle.

2. Point de vue évoqué par l'Université de Chicago : University of Chicago, vol 46, 1978-1979, pp. 288 et suivantes.

3. Millard E., 2000, "Legal realism and constraints", Conférence à la Faculté de droit de l'Universite de Copenhague, Danemark, septembre 2000, mis en ligne le 25 janvier 2007, consulté le 17 septembre 2010, URL : http://halshs.archives-ouvertes.fr/halshs-00126665/fr.

4. Delmas-Marty M., 2004, pp. 18-23.

5. Levi-Strauss C., 1983, pp. 59-62.

6. Ibid.

7. Ibid.

8. Le programme Paix et Gouvernance de l'Université de l'ONU s'efforce de promouvoir la paix durable et la bonne conduite des affaires publiques. L'humanité ne peut pas vivre sans peur quand plus d'un milliard de personnes continuent à vivre dans la servitude et la pauvreté. Cependant, il est aussi vrai qu'un environnement d'insécurité affaiblit les perspectives de 
croissance économique et de développement. Site de l'ONU, consulté le 15 octobre 2010, URL : http://isp.unu.edu/

9. Coutume étudiée par le géographe d'Heinrich Barth (1821-1865) lors de ses travaux sur une convention de partage du territoire. Cet exemple a aussi été cité par Claude Lévy Strauss, 1955, Triste tropique, Ed. Plon, Paris, p. 177 ; puis par Alexandre Adler, «Le royaume Moundang de Léré ", dans Tardits C. (dir.) Princes et serviteurs du royaume : cinq étude de monarchie africaines, Ed. Société d'ethnographie, Paris, 1987, pp. 137-170.

10. Malaurie J., 1974, pp. 3-29.

11. Voir l'article LeParisien.fr, "Une bombe nucléaire US perdue au Groenland », mis en ligne le 11 novembre 2008, URL: h http://www.leparisien.fr/international/une-bombe-nucleaire-usperdue-au-groenland-11-11-2008-306930.php. Voir aussi article du Nouvel Obs.com, « Une bombe nucléaire américaine perdue sous la glace ", mis en ligne le 11 novembre 2008, URL : http:// tempsreel.nouvelobs.com/actualite/monde/20081111.OBS0368/une-bombe-nucleaireamericaine-perdue-sous-la-glace.html

12. Le sororat est une des pratiques coutumières très anciennes qui aurait encore cours au Kasaï Oriental, une province de la République démocratique du Congo.

13. La common law est un système bâti essentiellement sur le droit jurisprudentiel, en opposition au droit civiliste ou codifié. C'est une conception d'origine anglaise qui marque la prééminence des décisions des tribunaux, la jurisprudence, le précédent.

14. « $\mathrm{Li}$ » est un rituel protégeant l'ordre social et impliquant donc la rectitude morale, autrement dit, « comportement correct ».Le sens donné au mot Li varie selon le contexte, mais se rapporte le plus souvent à l'idée de règle de conduite, de rite, ou de loi morale. Les penseurs chinois de l'antiquité décrivent ainsi les fonctions du Li : Sans ces règles, les devoirs mutuels du prince et du sujet, du supérieur et de l'inférieur, du père et du fils, du frère plus âgé et puîné, ne peuvent être déterminés. Le Li sert donc à établir et à maintenir les différences de statut. Et la justice doit être rendue conformément au Li.

15. He F., slnd, « Une longue marche vers l'Etat de Droit. La transformation du système judiciaire chinois ", Recherches en ligne, Etat de Droit et Justice, mémoire consulté le 2 novembre 2010, URL : http://www.dhdi.free.fr/recherches/etatdroitjustice/index.htm. De Bary W. M. T., 1998, Asian values and Human Rights. A Confucian Communitarian Perspective, Cambridge Mass., Harvard University Press, $196 \mathrm{p}$.

16. Mauss, 1950, pp. 144-279.

17. Pour Mauss, il s'agit de la puissance secrète et de la force mystérieuse.

18. Besnard (1998) retrace la correspondance scientifique, très riche, entre, Mauss, jeune chercheur et son oncle, Durkheim, conseiller et mentor.

19. Mauss, 1950, Op. cit., p. 29.

20. Ibid., pp. 72-184.

21. Ceux qui ne sont pas objets d'échange obligatoire (Mauss, 1924, p. 512). Cf. Baloma Spirits of the Dead, Jour. of the Royal Anthropological Institute, 1917.

22. Le mythe maori de Te Kanava (GREY, Polyn. Myth., Ed. Boutledge, p. 213) raconte comment les esprits, les fées, prirent l'ombre des «pounamu» (jades, etc.) exposés en leur honneur. Un mythe exactement identique à Mangaia (Wyatt GILL, Myths and Song front the South Pacifie, p. 257), relate la même chose à propos de colliers de disques de nacre rouge, et comment ils gagnèrent la faveur de la belle Manapa.

23. France Culture, le 25 septembre 2010, émission "Terre à terre ", diffusé à $07 h 05$, «Planète durable à Lyon: Des fleurs contre le pétrole», invité José Gualinga, représentant du peuple Kichwa d'Amazonie équatorienne et Corinne Arnould, de l'association Parole de Nature. 


\section{RÉSUMÉS}

Le titre de cette communication se veut quelque peu provocateur tant par les idées développées que par l'approche, anthropologique et géographique, retenue. Par l'anthropologie nous approcherons la question de la violence et de la concurrence au sein d'un même territoire et intrafirmes. Le choix de l'approche anthropologique associée à celle de la géographie permet de pallier un manque de prise en compte du «temps long» dont souffre souvent le développement en science économique. Or, la construction d'une analyse basée sur " l'intelligence économique » passe inévitablement par une prise en compte du «temps long», de la dimension humaine, des ressources naturelles, d'un territoire donné. L'anthropologie complète donc l'analyse du géographe en vue d'expliquer les effets de la violence économique sur un territoire: entre plusieurs populations, entre les firmes et les populations.

De ce fait, l'approche anthropologique montrera que l'ethnocentrisme est source de violence puisqu'il revient à appliquer sur un territoire, culturellement différent, un modèle de développement sans tenir compte de données anthropologiques et géographiques. Ce qui bien souvent engendre un épuisement des ressources, des conflits armés, des réseaux mafieux, des pollutions massives. Nous nous appuierons sur différents exemples évocateurs comme celui du Caucase, de la dispute du Groenland et de l'océan Arctique, ou encore l'exemple de l'introduction de la Perche du Nil dans le lac Victoria.

Après une approche anthropologique de la concurrence du monde des firmes qui permet de balayer le modèle de l'existence d'une concurrence pure et parfaite, la théorie du don et du contre-don développée par Marcel MAUSS (1924) est ensuite abordée. Enfin, par analogie, nous mettons en parallèle le lien entre le territoire et la firme. Cette culture du don et du contre-don permet d'illustrer le fonctionnement des firmes en vue du développement territorial et de souligner l'importance de l'observation sur la longue durée, indispensable à la compréhension du développement d'un territoire. En conclusion, le fonctionnement des firmes sur un territoire accroit les tensions, engendre la violence, car, en s'appuyant sur un modèle économique, elles oublient les dimensions culturelles et humaines qui sont des éléments constitutifs d'un territoire.

The title of this paper is somehow provocative both in the link between violence and the territorial development, and in the anthropological and geographical approach. By anthropology, I mean the link between violence and competition between firms within a territory. The anthropology as a social science and sibling of the geography permits to use an approach on a long run term which is not taken into account by the economy. Besides the idea of taking into account the notion of time, anthropology linked to geography covers the human aspect and the natural resources of a given territory. In addition to geography, anthropology reveals the notions of the effects of the economic violence on a territory among several populations, between firms and populations.

As a result, anthropology will show how ethnocentrism is a source of violence as we tend to use an economic pattern on a given territory with different habits and customs, without any notions of anthropology and geography. This lack of consideration leads to a shortage of resources, wars, corruption and high pollution. We will give you examples as the caucasian range of mountains, the conflict about Greenland and the Arctic ocean and the introduction of the Nil perch into the waters of the Victoria lake.

After an anthropological approach of the competitive market which reveals that a sound competition is not possible, we raise the theory of the "gift and counter-gift" explained by Marcel MAUSS (1924). This culture of "gift and counter-gift" is left aside by a business environment run by firms on a territory and economists who have their own vision of the 
economy. Therefore, we will point out that observation on a long run is essential and that the economic science has its limits. The economy has forgotten that time and history have played a major role in the development of a territory, too. As a conclusion, we can say that the firms increase tensions, cause violence for they rely on economic patterns forgetting about the cultural and human dimensions which are also necessarily part of a territory.

Der Titel dieses Artikels ist irgendwie provokant, sowohl im Zusammenhang mit Gewalt und der territorialen Entwicklung als im anthropologischen und geographischen Ansatz. Durch Anthropologie, handelt es sich um die Beziehungen zwischen Gewalt und Wettbewerb unter den Unternehmen innerhalb eines Territoriums. Die Anthropologie als Sozialwissenschaft und Geschwister der Erdkunde, erlaubt die Zeit zu berücksichtigen, die die Wirtschaft für die Entwicklung nicht genug beachtet. Neben der Erdkunde zeigt die Anthropologie, auf einem Gebiet, die Auswirkungen der wirtschaftlichen Gewalt zwischen mehreren Bevölkerungen, zwischen Unternehmen und Bevölkerung.

Es wird folglich gezeigt wie anthropologisches Ethnozentrismus eine Quelle der Gewalt ist, wie zu einer wirtschaftlichen Muster auf einem bestimmten Gebiet mit unterschiedlichen Sitten und Gebräuchen zu verwenden, ohne jegliche Gedanken an Anthropologie und Erdkunde zu berücksichtigen. Das führt oft zur Bedrohung der Ressourcennutzung, zu bewaffneten Konflikten, mafiösen Netzwerken und massiver Umweltverschmutzung. Wir werden verschiedene illustrative Beispiele nützen wie der Kaukasus-Konflikt, der Konflikt über Grönland und den Arktis und die Einführung des Nil Barschs in den Gewässern des Victoria Sees.

Eine anthropologische Betrachtungsweise des Wettbewerbs in der Firmenwelt zeigt, dass ein gesunder Wettbewerb nicht möglich ist. Nachher wird die von Marcel Mauss (1924) entwickelte Theorie der "Gabe und Gegengabe" erörtert. Diese Kultur der "Gabe und Gegengabe" illustriert wie die Unternehmen sich entwickeln, um neue Gebiete zu erobern und dass langfristige Beobachtungen eine wesentliche Bedeutung hat, um die Entwicklung des Territoriums zu verstehen.Zum Schluß, kann man sagen, dass die Unternehmen und ihr Prozess auf einem bestimmten Gebiet zu Spannunen und Gewalt führen, denn sie vergessen erst Wirtschaftorientiert die kulturellen und menschlichen Dimensionen, aus denen ein Territorium besteht.

\section{INDEX}

Keywords : anthropology, competition, development of a territory, firms, gift, violence Mots-clés : anthropologie, concurrence, développement territorial, don, firme, violence Schlüsselwörter : Anthropologie, die Entwicklung eines Gebietes, Geschenk, Gewalt, Konkurrenz, Unternehmen

\section{AUTEURS}

\section{ANTHONY TCHÉKÉMIAN}

Maître de conférences - Laboratoire CERPA - Université de Lorraine - Campus Lettres et Sciences Humaines - 3 place Godefroi de Bouillon - 54015 NANCY Cedex - anthony.tchekemian@univnancy2.fr

\section{RICHARD GAUTHIER}

Ingénieur-Economiste - Docteur en histoire du droit et des institutions - 20 avenue Foch - 54000 NANCY - rg20@hotmail.fr 